\title{
Efficient trigger signal generation from wasted backward amplified stimulated emission at optical amplifiers for optical coherence tomography
}

\author{
SeungTaek Kim ${ }^{1, a}$, HyungTae Kim ${ }^{1, b}$, YoungJune $\mathrm{Cho}^{1, \mathrm{c}}$, and Tae Joong Eom², \\ ${ }^{1}$ Smart Manufacturing Technology Group, KITECH, 35-3, HongCheon, IpJang, CheonAn, CungNam, 331-825, South Korea \\ ${ }^{2}$ Bio Photonics laboratory, APRI, GIST, 123, CheomDan-GwaGi RD., BukGu, GwangJu, 500-712, South Korea
}

\begin{abstract}
This paper propose an optical structure to generate trigger signals for optical coherence tomography (OCT) using backward light which is usually disposed. The backward light is called backward amplified stimulated emission generated from semiconductor optical amplifier (SOA) when using swept wavelength tunable laser (SWTL). A circulator is applied to block undesirable lights in the SWTL instead of an isolator in common SWTL. The circulator also diverts backward amplified spontaneous lights, which finally bring out trigger signals for a high speed digitizer. The spectra of the forward lights at SOA and the waveform of the backward lights were measured to check the procedure of the trigger formation in the experiment. The results showed that the trigger signals from the proposed SWTL with the circulator was quite usable in OCT.
\end{abstract}

\section{Introduction}

Since Huang's proposal for OCT[1], OCT has been applied to many biomedical systems, especially opthalmology[2]. Many of current technologies in OCT are based on SWTL. OCT systems using SWTL must synchronize optical signals with trigger signals which is mostly generated from a part of the output port of SWTL[3, 4]. However, this structure is basically inefficient and its output power is relatively insufficient, hence some studies reported that they adopt adopted additional booster optical amplifiers. Despite many efforts to increase the output power of SOAs, it is still worth developing efficient SWTL structures for OCT systems. One of the discarded elements in SWTL is backward lights caused by the SOA.

Therefore this study proposed how to use the backward lights and apply them to trigger generation for an efficient SWTL structure. The backward lights has been being disposed until now, however, this study utilizes the backward lights using a circulator, then most of output powers can be entirely applied to interference parts. The spectra using the proposed SWTL and the waveform of its filtered backward light were measured simultaneously to verify the procedure of trigger formation. The experimental results using the proposed structure show possibility to be applied for OCT systems.

\footnotetext{
ae-mail: htkim@kitech.re.k

be-mail: stkim@kitech.re.kr

ce-mail: choyj@kitech.re.kr

de-mail: eomtj@gist.ac.kr
}

\section{Basic Principles}

OCT systems using SWTL are conventionally composed of a SOA, isolators, Fabry Perot tunable band pass filter (FP-TBPF), dummy fiber (DF), a polarization controller (PoC), a variable coupler (VC), a circulator, arbitrary function generator (AFG), a current driver (CD) and an operating computer as shown in figure 1(a).When a SOA is powered using the $\mathrm{CD}$ as a sweep source, lights are generated from the SOA and pass isolator 2. In the VC, some lights are sent to the 10:90 coupler. Simultaneously, the other lights are directed to the $\mathrm{PoC}$ and transferred to the DF. Then, the FP-TBPF makes a light which has a specific wavelength and a narrow band depending on a voltage level applied by an AFG. The light from the FP-TBPF passes through isolator 1 , then feedbacked to the SOA as an input, which indicates optical amplification. This feedback procedure is continuously repeated using variable wavelength of lights, which is called SWTL. In other hand, the 10:90 coupler splits the inserted lights from the $\mathrm{VC}$ into two lights at the fixed split ratio there (1:9). A large part $(90 \%)$ of lights is transferred to interference parts and the operating computer which constructs OCT images and a small part (10\%) of lights is sent to clock generation block which is composed of the circulator 1 , the FBG, and the Photodiode and TTL signal generator. OCT structures proposed in many studies are based on SWTL, the light intensity for the operating computer is lowered due to passing couplers for light separation. In addition, most of backward stimulated lights at SOA has not been employed, hence intrinsically dissipates. Our structure is aimed at using the backward lights shown in figure 1(b) for generating trigger signals. The figure 1(b) shows that an additional circulator was adopted instead of isola- 
tor 1 in the SWTL shown in the figure 1(a) The circulator 2 is replaced to conduct the same function as the isolator 1 and transfer the backward light at the SOA to a trigger generation block. Hence the output light of SWTL can be entirely used to interference parts in OCT systems. In order to create trigger signals synchronized in a high speed digitizer, the PD+TTL signal generator was developed using a PIN photodiode, a trans-impedance amplifier and a comparator. Sampling using the trigger signals from the PD+TTL signal generator were also considered according to wavelength sweeping direction.

\section{Experiment}

The proposed structure of SWTL was implemented as shown in figure 2 and table 1 is its specification. The CD operates the SOA in constant current mode. Basically, the SOA makes bidirectional lights such as forward lights and backward lights. The backward light at SOA is inserted into port 2 (2) of the circulator 2 and then transferred to port 3 (3)) of the circulator 2. As shown in figure 1(b), the transferred light at port 3 (3)) of the circulator 2 are sent to the port 1 (1) of the circulator 3 and then transferred to the port 2 of the circulator 3 . Then, they meet the FBG with its center wavelength of $1284.6 \mathrm{~nm}$. Most of backward lights pass through the FBG and is dissipated. However, the lights with the wavelength of $1284.6 \mathrm{~nm}$ are only reflected in the FBG and feedbacked into the port 2 (2) of the circulator 3 again. Then, at the port 3 of the circulator 3, the filtered lights go through the photodiode and TTL signal generator. The photodiode converts the sent lights to analog current signals and the trans-impedance amplifier also makes the analog voltage signals from the analog current signals. Finally, the comparator changes the generated analog voltage signal into digital logic level signals. The photodiode and the LV-TTL signal generation circuit make trigger signals. The resonance frequency of the FP-TBPF is about $52.6 \mathrm{kHz}$ that decides the length of dummy fibers as $3.9 \mathrm{~km}$. The photodiode and the LV-TTL signal generation circuit make trigger signals for a high speed digitizer.

The spectra of the proposed SWTL were measured using an optical spectrum analyzer (OSA). The measurement was conducted at 4 points: forward SOA, backward light, and reflected light by fiber Bragg grating (FBG). Waveform of trigger signals were time-domain and monitored using an oscilloscope.

\section{Results}

The spectra measured using the OSA and the trigger signals acquired using the oscilloscope are shown in figure 3 . The output spectrum of the proposed SWTL was measured before interference parts in figure 1(b) and was shown in figure 3(a) when operation current applied to the SOA was $300 \mathrm{~mA}$. The spectral range was from $1271.4 \mathrm{~nm}$ to $1345.4 \mathrm{~nm}$, then the bandwidth of the proposed SWTL was about $74 \mathrm{~nm}$. While measuring, the resolution bandwidth and the sensitivity measured by the OSA was $1 \mathrm{~nm}$ and $60 \mathrm{dBm}$, respectively. Figure 3(b) shows the spectrum of backward lights which is measured at the port 3 of the circulator 2 when keeping the same driving current level to SOA. The extracted backward light was feedbacked into the trigger signal generation block. Comparing figure 3(a) with figure 3(b), the spectrum of extracted backward light from the circulator 2 represented slightly narrower bandwidth and smaller amplitude than the output spectrum of the SWTL. The narrow-banded signal in the OSA sweeps a flat plateau in the spectrum from $1280 \mathrm{~nm}$ to $1340 \mathrm{~nm}$. For the functional verification of the FBG, the spectrum of the reflected light at the FBG was also measured at the port 3 of the circulator 3 and is shown in figure 3(c). From the maker 1's position in figure 3(c), the peak wavelength of the filtered light at the FBG is $1284.6 \mathrm{~nm}$. The reflected lights at the port 3 of the circulator 3 were sent to the PD+TTL signal generator where optical signals were converted into electrical logic signals. Their waveforms are acquired using the oscilloscope and shown in figure 3(d). The first trigger signal (1) comes from downward wavelength sweeping from $1354.4 \mathrm{~nm}$ to $1271.4 \mathrm{~nm}$ and the second signal (2) from upward wavelength sweeping from $1271.4 \mathrm{~nm}$ to $1354.4 \mathrm{~nm}$ in the figure 3(d). In the same manner, the third comes from downward wavelength sweeping and the fourth from upward wavelength sweeping. In the other hand, there are two different intervals formed in figure 3(d). One of them is a yellow solid horizontal line (b) which means the round trip time from $1284.6 \mathrm{~nm}$ to the maximum sweeping wavelength. The other is a yellow dashed horizontal line (@) which means the round trip time from $1284.6 \mathrm{~nm}$ to the minimum sweeping wavelength. One of the two intervals has to be selected as a trigger source for image processing. The first (1) and third signal (3) of the four must be chosen as a trigger signal when wavelength of the SWTL decreases but the second (2) and the fourth (4) be selected when wavelength of the SWTL increases. For an example, if the second and the forth signals are selected, the analog-todigital converter module has to keep and to save the data acquired at from the half of (a) to the half of (b) every time when it receives the trigger signals. From the timing analysis shown in the figure 3(d), the modulation frequency of driving current at SOA can be determined. All these experimental results imply that the trigger signals generated from the backward light has regularity.

\section{Conclusion}

This study proposed how to use backward lights in SOA and apply the back lights to generate the trigger signal replacing an isolator by a circulator. The backward lights have been disposed in previous studies, however, the experiment results show that the trigger signal using the backward lights is useful for synchronizing image data.

\section{References}

[1] D. Huang, E. A. Swanson, C. P. Lin, J. S. Schuman, W. G. Stinson, W. Chang, M. R. Hee, T. Flotte, K. Gregory, 


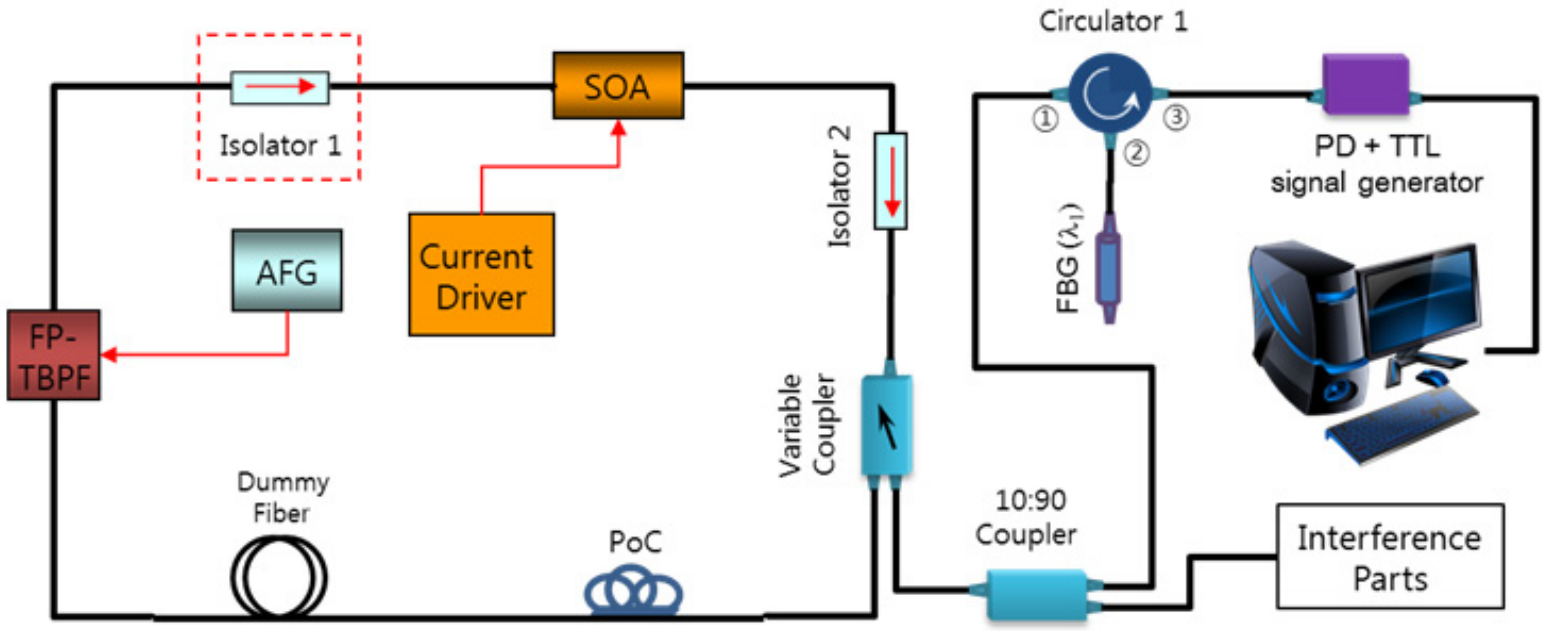

(a)

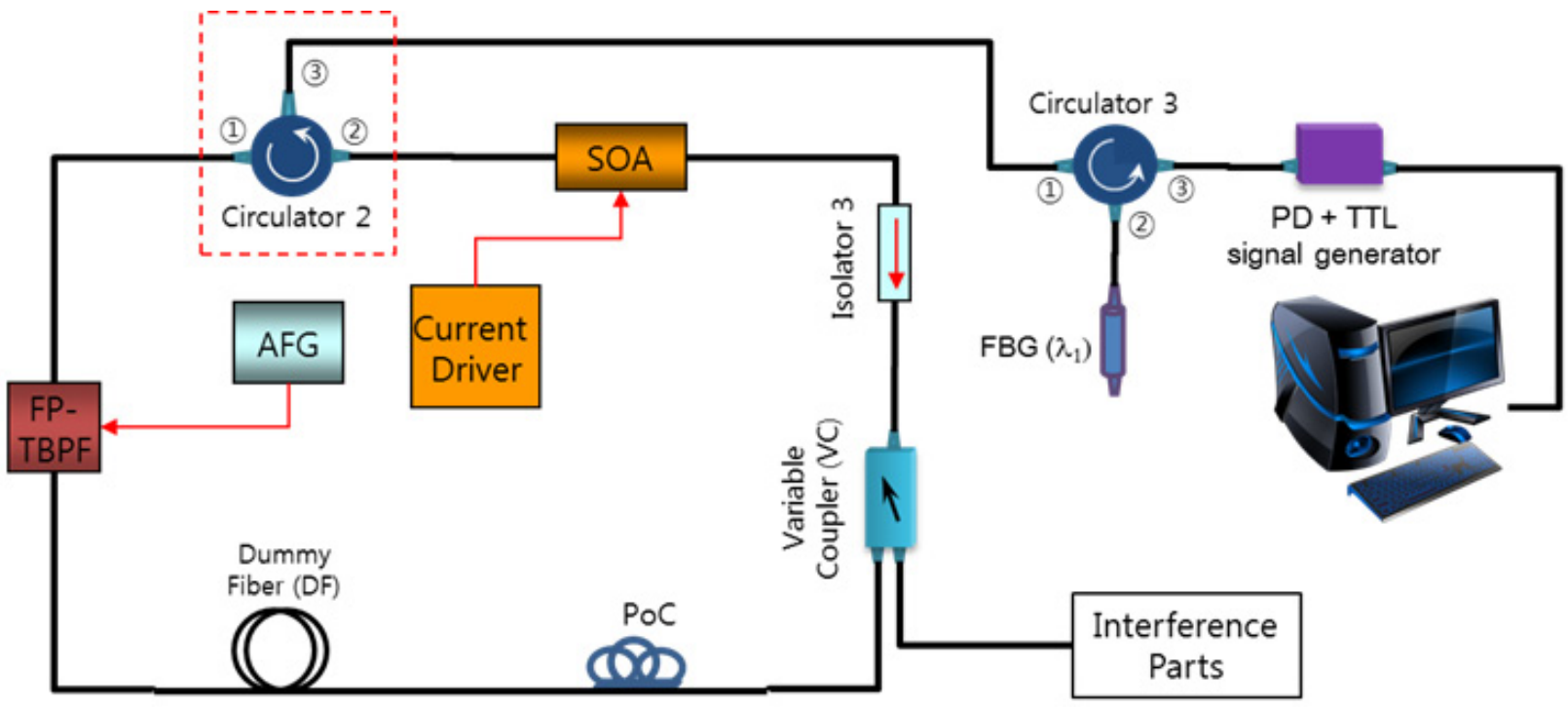

(b)

Figure 1. Swept wavelength tunable lasers (a) a general structure using an isolator (b) a proposed structure using a circulator

Table 1. Specification of the experiment system

\begin{tabular}{llll}
\hline components & manufacturer & model & specification \\
\hline FP-TBPF & Lambda Quest & 1300nm & Gain $<23 \mathrm{~dB}$, Spectral range 40-160nm \\
PoC & Fiberpro & & Center wavelength 1300nm \\
VaC & Fiberpro & & Center wavelength 1300nm \\
SOA & QPhotonics & QOSA-1300 & Gain <30dB, 1290-1330nm \\
CD & Notice & Dual SOA & Maximum frequency 1MHz, power 1.0A \\
Circulator & Thorlabs & CIR-1310-50-APC & Spectral range 1280-1400nm \\
AFG & Tektronics & AFG3252C & Maximum frequency 240MHz \\
Oscilloscope & Tektronics & TDS5104B & Maximum sampling rate 1GHz \\
OSA & HP & 86140B & Spectral range 600nm-1700nm \\
\hline
\end{tabular}




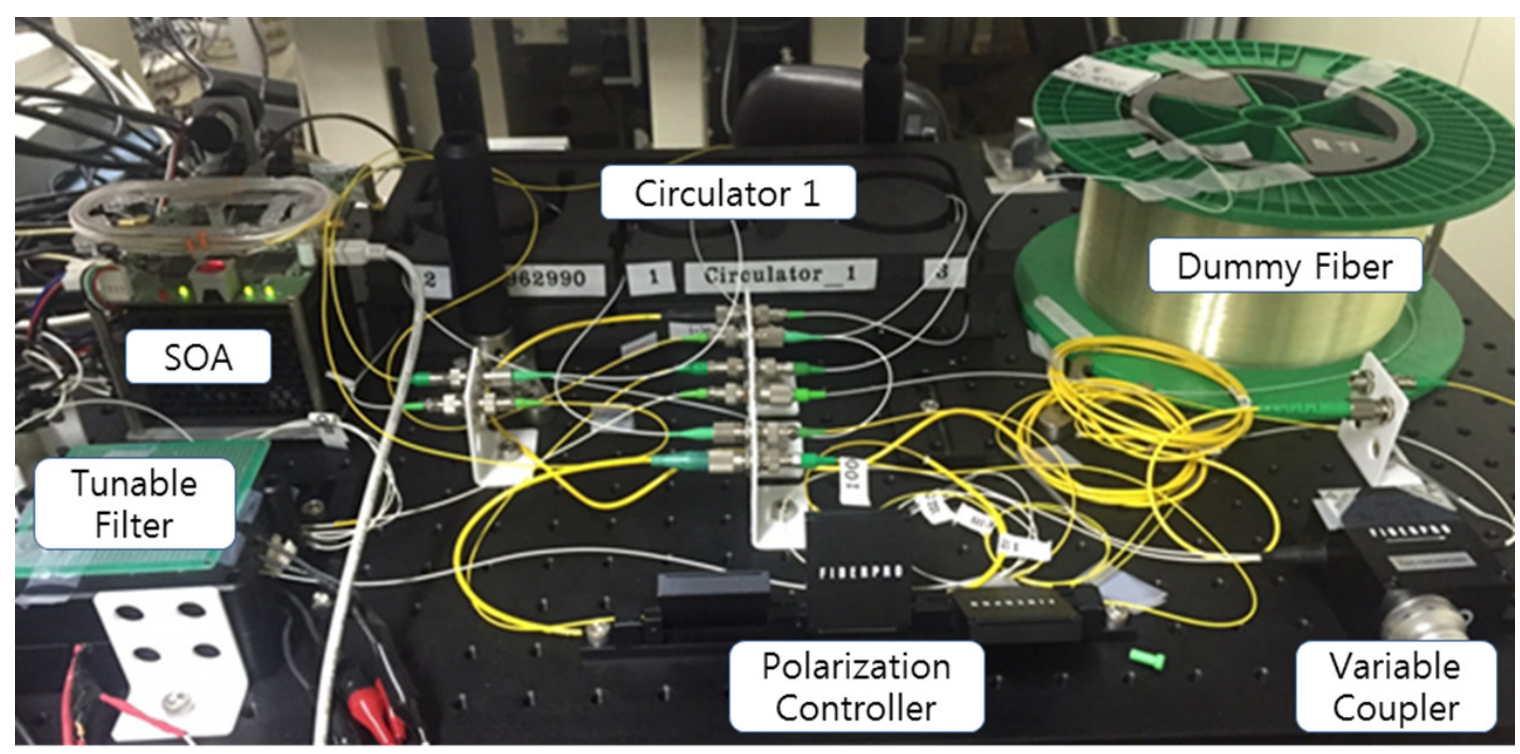

Figure 2. photo of the proposed SWTL using a circulator

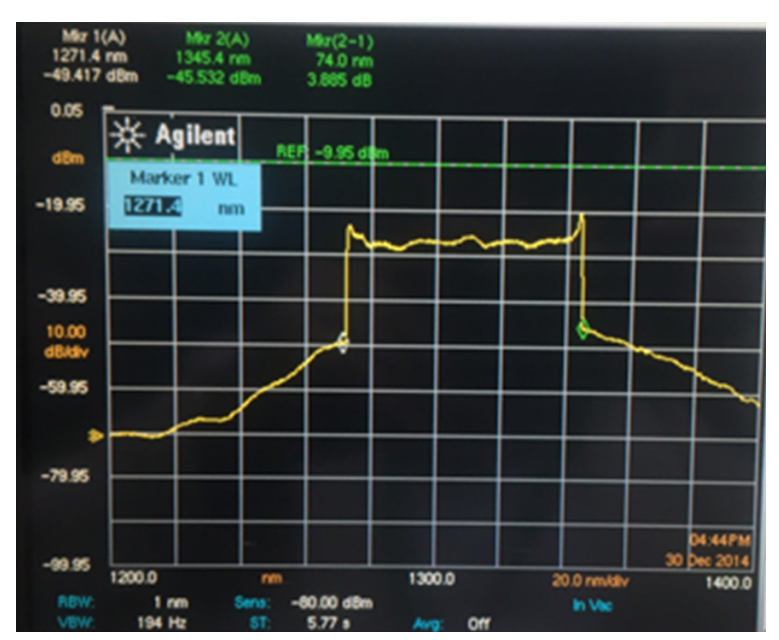

(a)

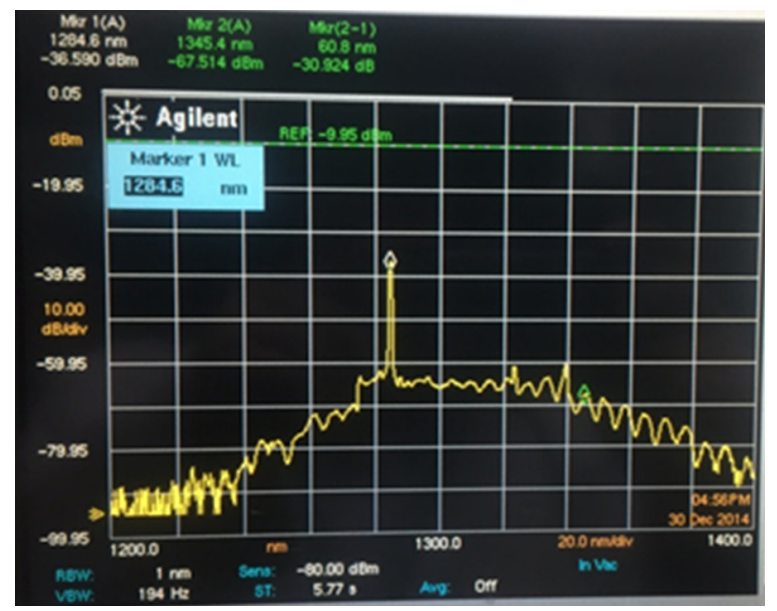

(c)

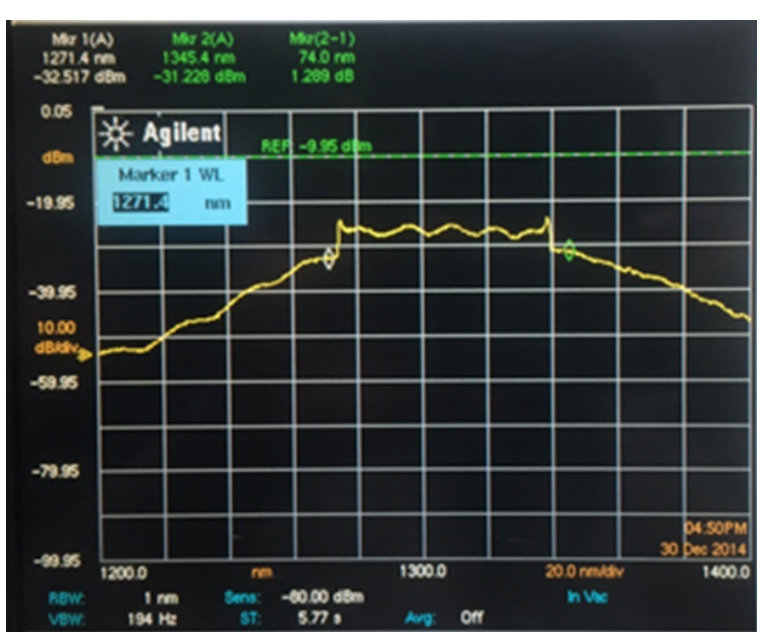

(b)

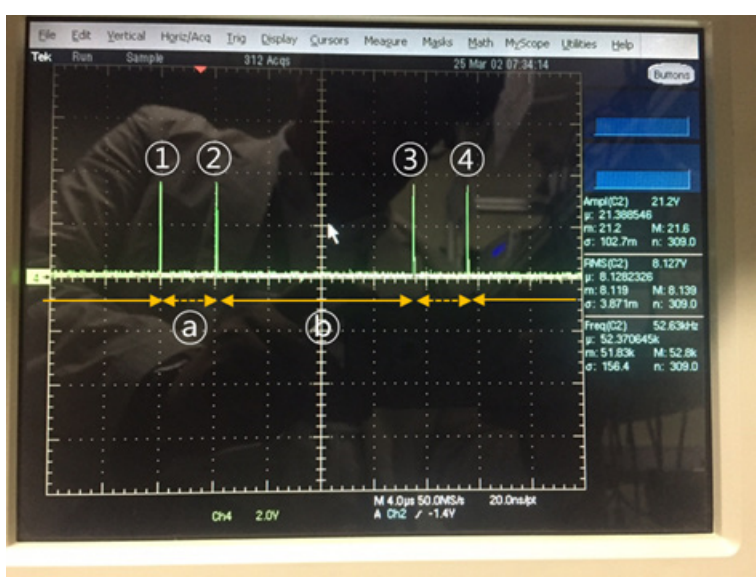

(d)

Figure 3. Spectra measured by an OSA (a) forward SOA (b) backward lights (c) Bragg grating and Waveforms measured by an oscilloscope (d) trigger signals 
C. A. Puliafito, and J. G. Fujimoto, Science 254, 11781181 (1991)

[2] W. Drexler and J. G. Fujimoto, Optical Coherence Tomography (Springer, Berlin, 2008) 1-14

[3] F. D. Nielsen, L. Thrane, J. Black, K. Hsu, A. Bjarklev and P. E. Andesrson, Proceeding of SPIE 5861, 58610H (2005)
[4] S. H. Yun, C. Boudoux, M. C. Pierce, J. F. de Boer, G. J. Tearney, and B. E. Bouma, IEEE Photonics Technology Letter, 16 293-295 (2004)

[5] S. Shin, U. Sharma, H. Tu, W. Jung and S. A. Boppart, IEEE Photonics Technology Letter, 22 1057-1059 (2010) 